\title{
Percepción del Impacto de la Covid-19 en los Profesionales de la Educación Social que Trabajan con Menores
}

\author{
Perception of the Impact of Covid-19 on Social Education \\ Professionals Working with Minors
}

\author{
Alejandro Martínez-Pérez * \\ Fernando Lezcano-Barbero \\ Universidad de Burgos, España
}

\begin{abstract}
La Covid-19 ha producido un impacto en todo el mundo y el sector educativo no se ha visto exento del mismo. La acción socioeducativa en España tiene como referente a los profesionales de la educación social que desarrollan su actividad profesional en diferentes ámbitos y el sector de la infancia y la juventud es uno de los predominante. Con este artículo pretendemos conocer la percepción del impacto que la crisis ha tenido entre los profesionales de la educación social que trabajan con menores. El enfoque metodológico es mixto, mediante un cuestionario creado ad hoc, autoadministrado por internet. La muestra es de ámbito estatal (España), 462 profesionales. Los resultados reflejan como la actividad laboral ha sido declarada esencial y no ha supuesto modificaciones contractuales, pero sí laborales. Asimismo, la carga de trabajo se ve aumentada y la posibilidad de cumplir con los objetivos planteados no es demasiado elevada. Abordamos también la satisfacción laboral y sobre las herramientas y competencias digitales de los menores con los que trabajan estos profesionales. Comparamos los datos con resultados anteriores y concluimos la necesaria dotación de mayores recursos personales y materiales para los profesionales y servicios y la necesidad de proveer de herramientas y competencias digitales entre los menores usuarios.
\end{abstract}

Descriptores: Educación social; Infancia; Juventud; Educación; Covid-19.

Covid-19 has had an impact worldwide and the education sector has not been spared. Social and educational action in Spain has as its reference the professionals of social education who develop their professional activity in different areas and the sector of childhood and youth is one of the predominant ones. With this article, we aim to understand the perception of the impact that the has had on social education professionals working with children. The methodological approach is mixed, using a questionnaire created ad hoc, self-administered through the Internet. The sample is nationwide (Spain), 462 professionals. The results reflect how the work activity has been declared essential and has not involved contractual modifications, although there have been labor changes in the development of the work. Likewise, the workload is increased and the possibility of meeting the objectives set is not too high. We also address issues relating to job satisfaction and the digital tools and skills of the minors with whom these professionals work. We compared the data with previous results and concluded the necessary provision of more personal and material resources for professionals and services and the need to provide digital tools and competences among minors.

Keywords: Social education; Childhood; Youth; Education; Covid-19.

*Contacto: alejandromp@ubu.es

ISSN: 2254-3139

www.rinace.net/riejs/

revistas.uam.es/riejs
Recibido: $\quad 3$ de mayo 2020

$1^{\text {a }}$ Evaluación: 25 de junio 2020

$2^{\text {a }}$ Evaluación: 20 de julio 2020

Aceptado: 25 de julio 2020 


\section{Introducción}

La Covid-19 ha irrumpido en el año 2020 en todo el mundo provocando una profunda crisis sanitaria y económica, así como cambios radicales en la sociedad y en la manera relacionarnos y comunicarnos.

En el ámbito educativo, en España, se han suspendido las clases presenciales que, al menos, hasta final de curso se llevarán a cabo de manera telemática y también ha afectado a otros programas educativos de infancia y juventud en el ámbito de la educación no formal.

En nuestro artículo, tras una revisión de la literatura, nos proponemos analizar el impacto que este virus ha tenido entre los educadores y educadoras sociales que trabajan con menores.

\section{Revisión de la literatura}

\subsection{La educación social en España}

Para Pérez Serrano (2003) el surgimiento de la Pedagogía Social es el resultado de la confluencia de circunstancias históricas, sociales y políticas en Europa. Para Tiana (2014) la educación social es la respuesta que permite atender las necesidades y demandas sociales debidas a los cambios producidos a lo largo de la historia; recoge experiencias previas a la consolidación del campo profesional y la disciplina académica.

Ortega, Caride y Úcar (2013) plantean la educación social en España como la confluencia de tres corrientes de pensamiento y acción que llegan a lo largo del siglo XX: la corriente alemana, con una preocupación por humanizar la vida comunitaria y centrada especialmente en los procesos de inadaptación de niños y jóvenes; la corriente francófona, con un matiz más sociocultural y dirigida a la atención de los problemas sociales y comunitarios; y la corriente anglosajona, con un carácter más pragmático y empirista, que analiza la realidad desde la Sociología de la Educación y con intervenciones centradas en los servicios sociales con un enfoque más asistencialista o terapéutico.

En los momentos anteriores a la creación de la titulación académica existe una apertura hacia las variables sociales y culturales en el ámbito educativo, con un despliegue en lo espacial, nuevos ámbitos de intervención educativa, y en lo temporal -educación permanente y nuevos destinatarios de esta- advertido por organismos internacionales como la UNESCO o el Consejo de Europa. En estos años se produce un aumento de la demanda social de estas acciones y un surgimiento de múltiples y heterogéneas prácticas en educación social (Ventosa, 1989).

El 10 de mayo de 1991 el Boletín Oficial del Estado (BOE) publica el Real Decreto 1420/1991 con el que se establece el título de Educación Social en España. Será una diplomatura universitaria, con una carga mínima de 180 créditos y tres años de duración. Se consolida así la educación social como una titulación dirigida a la "formación de un educador en los campos de la educación no formal, educación de adultos (incluidos los de la tercera edad), inserción social de personas desadaptadas y minusválidos, así como en la acción socio-educativa" (RD 1420/1991, p. 1).

Posteriormente, debido a la adaptación al Espacio Europeo de Educación Superior (EEES) de las titulaciones universitarias se ha transformado en el Grado en Educación Social que 
puede cursarse actualmente. Se trata de una titulación universitaria con una carga de 240 créditos europeos (ECTS) y una duración de cuatro años.

Es complicado realizar un análisis unificado de la educación social fuera de España ya que, tanto a nivel formativo como laboral, encontramos una gran dispersión. Sólo en Europa se cuentan más de 17 titulaciones diferentes y distintas clasificaciones profesionales y nomenclaturas entre países (ANECA, 2005; Calderón, 2013).

\subsection{El trabajo con menores desde la educación social}

El ámbito de intervención en la educación social es muy extenso, recorriendo una amplia variedad de colectivos en riesgo de exclusión social (Martínez-Pérez y Lezcano-Barbero, 2020). Esa amplia variedad no diluye la enorme importancia que tiene la intervención relacionada con menores. La educación social especializada ha estado presente mucho antes de crearse la titulación universitaria, como una práctica que traslada un enfoque educativo al trabajo social. No obstante, se pueden apuntar acciones con infancia y adolescencia desde una doble perspectiva: preventiva y reeducadora; pudiendo encontrar realidades que abarcan centros de protección y reforma de menores, educación de calle, residencias infantiles, casas de juventud, centros escolares, centros de tiempo libre, etc. (López Noguero, 2005).

El estudio de Cacho Labrador en Cataluña (1999) recoge como una de las seis áreas de trabajo del educador y educadora social la de los servicios sociales de base e infancia y adolescencia. Posteriormente, Vallés Herrero (2011) realiza un estudio a nivel estatal en tomando como referencia las líneas definidas por Cacho Labrador y el Practicum de la UNED para la titulación y establece cinco áreas de actuación: el área trabajo con menores es la más representativa de su muestra con un $45 \%$ de educadores y educadoras sociales que trabajan en la misma, principalmente en centros de reforma y equipamientos de protección.

Más recientemente, el trabajo de Álvarez Fernández (2017) realiza una clasificación en ámbitos de intervención en la que atendiendo al criterio de edad encontramos representadas la infancia y la juventud. Asimismo, la propuesta de Eslava Suanes (2019) recoge una clasificación en torno a colectivos, ámbitos y tareas, siendo la infancia y la juventud dos de colectivos representados. En el Catálogo Nacional de Ocupaciones, elaborado por el Instituto Nacional de Estadística (INE), se puede observar como entre los campos de acción recogidos para el educador y educadora social están la infancia y adolescencia (Martínez-Pérez y Lezcano-Barbero, 2020).

Finalizamos este apartado con las nuevas funciones que están surgiendo para la educación social dentro del marco del sistema educativo y que se añaden a las tradicionales. Aún es una realidad incipiente ya que apenas un 3\% de titulados son contratados en el ámbito reglado (ANECA, 2005).

Sierra y otros (2017) confirman la introducción del educador y educadora social en los centros escolares y recogen tres escenarios diferenciados en la actualidad en España, siguiendo la propuesta de López Zaguirre (2013): comunidades autónomas que adscriben a educadores y educadoras sociales en los centros a través de la normativa (Extremadura, Castilla - La Mancha y Andalucía), proyectos integrados desde los servicios sociales con funciones en centros escolares y proyectos concretos liderados por educadores y educadoras sociales en centros escolares, impulsados bien en solitario o desde el ámbito privado, o bien por la propia administración. Así, más allá de las comunidades que ya lo 
integran en su normativa, Galán Carretero (2019) recoge experiencias en Aragón, Islas Baleares, Islas Canarias o Galicia.

En las comunidades autónomas con implantación, los educadores y educadoras sociales realizan funciones relacionadas con la convivencia, el absentismo, la integración y la inclusión, la participación, el acompañamiento y seguimiento de alumnado en situación de riesgo y dificultad y otros programas socioeducativos. Asimismo, tienen una función dinamizadora entre escuela y familias y de coordinación con los recursos sociales (RuízRomán, Molina y Alcaide, 2019; Sierra et al., 2017; Vila, Cortés y Martín, 2020).

\subsection{Repercusión de la Covid-19}

En diciembre de 2019 se notifican varios casos de neumonía viral desconocida en la región de Wuhan, en China. En enero de 2020 se detecta como un nuevo tipo de coronavirus capaz de afectar y transmitirse entre humanos, denominado Covid-19. El 11 de marzo de 2020, según la Organización Mundial de la Salud (OMS), se confirman 80.955 casos en China y 37.367 a nivel mundial, fuera de China, estando España entre los países afectados por el nuevo virus (Isaifan, 2020). A finales de abril el Gobierno de España recoge un total de 203.715 casos de Covid-19 en nuestro país y un número de defunciones confirmadas de 15.873 (Centro Nacional de Epidemiología, 2020). En la última semana del mes (24-30 de abril), según las actualizaciones diarias del Ministerio de Sanidad, la media de confirmados diarios era superior a los 2.000 casos y las defunciones diarias se situaban por encima de los 322 casos.

El 11 de marzo de 2020 la OMS eleva la emergencia pública a pandemia mundial y el 14 de marzo de 2020, el Gobierno de España mediante el Real Decreto 463/2020, declara el estado de alarma para la gestión de la crisis sanitaria derivada de la Covid-19. En el ámbito educativo tiene importantes repercusiones como la suspensión de la actividad formativa presencial en todos los centros y etapas, ciclos, grados, cursos y niveles de enseñanza.

Posteriormente, el 29 de marzo, mediante el Real Decreto-ley 10/2020, el Gobierno establece un permiso retribuido obligatorio a los trabajadores de todos los sectores no esenciales que no puedan realizar su actividad laboral mediante teletrabajo. Quedarían exentas de este decreto las actividades educativas regladas en todas las etapas, al realizarse mediante tele-formación, y también las actividades consideradas esenciales en dicha normativa, entre las que se encuentran los centros o servicios que atienden a menores.

Organismos internacionales, como la UNESCO, alertan que el cierre temporal de las escuelas provoca altos costos sociales y económicos tales como la interrupción del aprendizaje, la suspensión de la alimentación en niños que reciben la comida de manera gratuita o a costo inferior en las escuelas, insuficiencias en el cuidado de niños o aumento de la presión en los centros escolares (UNESCO, s.f.).

UNICEF (2020), en un reciente informe, aborda los riesgos potenciales de la pandemia a nivel educativo en tres niveles:

- Entorno familiar: enfermedad, aumento de la presión y el miedo, reducción de resultados y aumento del absentismo escolar, estrés e impacto emocional que podría producir abandono escolar.

- En centros educativos: ausencia del profesorado por enfermedad o cuidado de familiares, estigmatización de niños que han sufrido la enfermedad, dificultades 
para cumplir con las medidas y estándares sanitarios (especialmente en centros con elevado número de alumnado), aumento de la violencia a grupos vulnerables.

- En el sistema educativo: aumento del retraso administrativo, impacto en el desarrollo educativo, posible disminución de rendimiento debido a la gestión, necesidad de contar con mecanismos de información y seguimiento actualizados.

Respecto a los centros y servicios que trabajan con menores, hemos podido conocer algunas noticias como la actuación de Médicos del Mundo (2020) en centros de tutela en Melilla, casos de incertidumbre y miedo entre menores tutelados (Fernández, 2020) y también entre trabajadores y direcciones de los centros.

La repercusión de la pandemia tiene un efecto más allá del inminente, con implicaciones psicológicas entre la población como el incremento de las emociones negativas, el aumento de la sensibilización hacia los riesgos y la disminución de emociones positivas (Li et al., 2020). Entre los jóvenes, un estudio realizado en China (Liang et al., 2020) indica como este tipo de situaciones pueden afectar a la salud mental del colectivo, asociándose, además, factores como el nivel educativo en la aparición de trastornos y angustia.

Otro de los riesgos ante la situación generada por la crisis sanitaria derivada de la Covid19 es el peligro de aumento de la violencia intrafamiliar que puede afectar a los menores. (UNICEF, 2020; Usher et al., 2020).

Respecto a la situación de los profesionales socioeducativos queremos destacar algunos trabajos que se refieren a la satisfacción laboral y su implicación en el desempeño laboral. En primer lugar, el estudio de Van Aerden y otros (2016), a nivel europeo, que indica que aspectos tales como la imprevisibilidad de horarios, las largas jornadas laborales y las horas extraordinarias no remuneradas afectan en el nivel de satisfacción laboral de los trabajadores.

En segundo lugar, la investigación de Calitz, Roux y Strydom (2014) entre trabajadores del sector social que apunta como las condiciones físicas del trabajo o las prácticas administrativas de la empresa pueden afectar a la satisfacción laboral. En el mismo sentido incide el estudio de Gómez, Alonso y Llamazares (2018) que expone como las consecuencias de la baja satisfacción laboral en el ámbito de los servicios sociales pueden llevar al agotamiento, la disminución de los niveles de rendimiento y una menor calidad en la prestación de servicio.

En último lugar el trabajo de Acker (2018), que nos indica como la satisfacción laboral es uno de los elementos que influyen el desempeño de los profesionales que trabajan en el ámbito de los servicios sociales.

El objetivo de nuestro trabajo es conocer el impacto que la crisis derivada de la Covid-19 ha tenido entre los profesionales de la educación social que trabajan con menores.

\section{Método}

El enfoque metodológico es mixto y aplicado de manera secuencial, con una primera fase prioritaria de corte cuantitativo y una segunda de corte cualitativo que nos permite obtener información complementaria (Creswell, 2015). La herramienta utilizada para la recopilación de los datos ha sido el cuestionario Covid_Edusocial, con preguntas cerradas y abiertas, y se ha realizado un muestreo, entre el 16 y 26 de abril de 2020, a través de la 
técnica de bola de nieve (Cresswell, 2012) para lo que se ha difundido el instrumento en internet; llamamos la atención especialmente de la colaboración de los Colegios Profesionales de Educación Social y en las redes sociales WhatsApp, Facebook, Twitter, Instagram y LinkedIn. El análisis cuantitativo ha sido realizado mediante el programa SPSS (Licencia de la Universidad de Burgos). Para la pregunta abierta se ha realizado un planteamiento inductivo mediante la inmersión en texto y la creación de categorías utilizando el software OpenCode. Se recogen, asimismo, algunas de las respuestas más relevantes de las diferentes categorías indicando el sexo, la comunidad autónoma en que trabaja y los años de experiencia laboral en educación social de la persona que las realiza.

\section{Instrumento}

El cuestionario Covid_Edusocial ha sido creado ad hoc, ante la inexistencia de una herramienta específica para esta situación, y sigue la estructura recogida en la Figura 1. Se trata de un instrumento anónimo y autoadministrado. Consta de 52 preguntas, 3 abiertas y 49 cerradas, distribuidas según el perfil de las personas participantes en un tronco común para todo el conjunto de la muestra y tres partes diferenciadas: para quienes realizan trabajo presencial, teletrabajo o se encuentran en situación de desempleo.

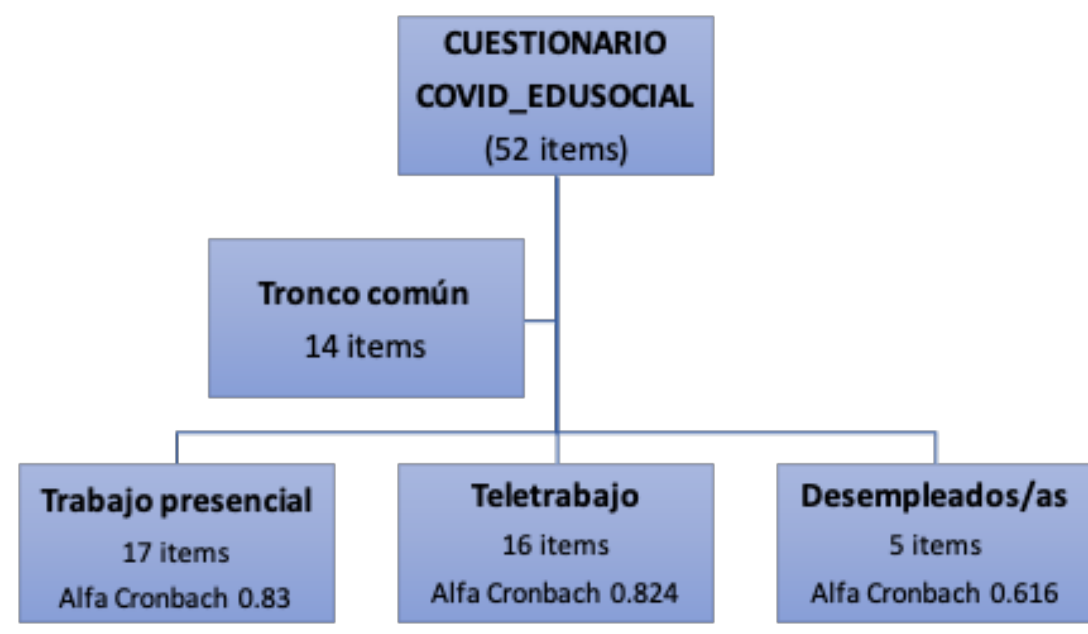

Figura 1. Estructura del cuestionario Covid_Edusocial

Fuente: Elaboración propia.

La parte común se compone 14 ítems, 11 preguntas cerradas sobre datos sociolaborales (sexo, edad, colectivo, relación laboral) y sobre la afectación del estado de alarma a su situación laboral, así como tres preguntas abiertas al final del cuestionario. Para el diseño de esta parte hemos adaptado el cuestionario EdSocEval_V2 (Martínez-Pérez y LezcanoBarbero, 2020) y tomado como referencia la diferente normativa del Gobierno para hacer frente a la crisis sanitaria.

La parte dirigida a quienes trabajan presencialmente se compone de 17 ítems tipo Likert con valoraciones acerca de cuestiones relacionadas con el desempeño de su trabajo: posibilidad de cumplimiento de objetivos, condiciones, satisfacción...; así como sobre la información la información recibida por parte de los empleadores y la disposición o no de Equipos de Protección Individual (EPIs). En el desarrollo de este apartado hemos tomado como referencia las recomendaciones del Ministerio de Sanidad (2020) en la guía Medidas para la prevención de contagios del Covid-19. Buenas prácticas en los centros de trabajo en su versión del 11 de abril de 2020. 
La parte dirigida a quienes realizan teletrabajo se compone de 16 ítems tipo Likert acerca del desempeño profesional: medios disponibles para los profesionales y los usuarios/as del servicio, competencia digital, cumplimiento de objetivos, satisfacción...

El apartado destinado a personas desempleadas se compone 5 ítems tipo Likert sobre el contacto con compañeros, directivos, empresa y usuarios, así como sobre las expectativas de retomar la actividad una vez se supere esta situación.

Se ha realizado un análisis de fiabilidad del cuestionario mediante el cálculo de la consistencia interna a través del Alfa de Cronbach en cada una de sus partes. Los resultados pueden considerarse buenos en el caso de actividad presencial $(0,83)$ y telemático $(0,824)$ y aceptables en el caso de las personas en desempleo $(0,616)$ según las medidas habitualmente utilizadas (Taber, 2017).

\section{Muestra}

La muestra está compuesta 462 profesionales que desarrollan su trabajo con menores, 351 $(76 \%)$ son mujeres, 110 hombres $(23,8 \%)$ y 1 persona nos indica la opción otros en este ítem $(0,2 \%)$. La edad media es de 37,03 años y varía entre los 20 años de mínimo y los 61 años de máximo. La mediana es 36 y la moda 32. Los años de experiencia laboral abarcan entre el mínimo de 1 y el máximo de 47, con una media de 10,87; una mediana de 10 y una moda de 1. Hay representación de todas las Comunidades Autónomas, excepto de Asturias, con diferente tasa de respuesta (figura 2). Respecto a su relación con la educación social predominan los diplomados $(\mathrm{n}=208 ; 45 \%)$, seguido de los graduados $(\mathrm{n}=157$; $34 \%)$, en tercer lugar, las personas habilitadas por algún colegio profesional $(\mathrm{n}=67$; $14,5 \%)$ y, en último lugar, quienes manifiestan tener otra relación con la educación social $(\mathrm{n}=30 ; 6,5 \%)$.

La mayoría de la muestra se compone de personas que trabajan para entidades del tercer sector sin ánimo de lucro $(\mathrm{n}=265 ; 57,4 \%)$, una tasa del $26,8 \%(\mathrm{n}=124)$ trabajan en la administración pública, un $13 \%(\mathrm{n}=60)$ lo hacen en empresas privadas, 2 personas $(0,4 \%)$ son autónomas y $11(2,4 \%)$ se encuentran actualmente en desempleo. Respecto a sus contratos el $12 \%(\mathrm{n}=57)$ se declara funcionario, un $53,7 \%(\mathrm{n}=248)$ trabaja con contrato indefinido en la empresa privada o como laboral en la administración pública, un 24,7\% (n $=114$ ) con contrato eventual y un $5 \%$ lo hace con contrato fijo-discontinuo. El porcentaje restante (20\%) tiene otro tipo de relación laboral (autónomos, socios de cooperativas...) o está en desempleo (figura 2).

Respecto a los ámbitos de trabajo, en su mayoría desarrollan su labor en recursos de atención a menores, bien de tutela o de reforma $(n=394 ; 85,3 \%)$, seguido de quienes trabajan como educadores/as sociales en el ámbito escolar $(\mathrm{n}=38 ; 8,2 \%)$. La muestra se compone también de 12 personas $(2,6 \%)$ que trabajan con menores dentro del ámbito de familia, en Puntos de Encuentro Familiar (PIF), Equipos de Tratamiento Familiar (ETF) u otros recursos análogos. Un total de $9(1,9 \%)$ personas trabajan con menores en el ámbito de la dependencia, la salud mental o la atención a la discapacidad. $3(0,6 \%)$ personas en educación de calle o medio abierto, $2(0,4 \%)$ en justicia juvenil y 4 personas $(0,9 \%)$ en otros ámbitos como puntos de información juvenil o técnicos de juventud en administraciones.

Para el análisis de los datos respecto a los ámbitos se han agrupado en dos categorías: educación formal, con los educadores/as sociales que trabajan en centros escolares ( $8,2 \%)$ 
y educación no formal con todas aquellas personas que llevan a cabo su trabajo en entornos fuera del sistema educativo reglado $(91,8 \%)$.

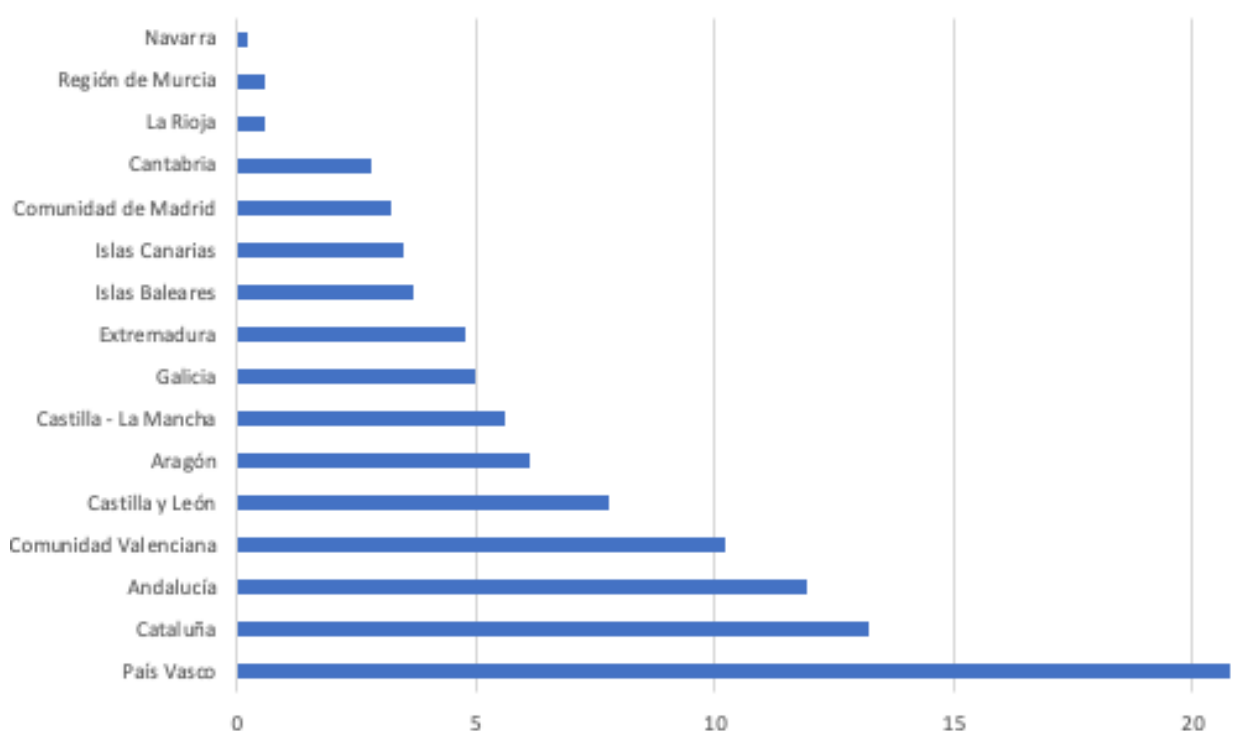

Figura 2. Distribución de la muestra por Comunidades Autónomas Fuente: Elaboración propia.

\section{Resultados}

\subsection{Impacto en la situación contractual y laboral}

Respecto a la contratación, en la mayoría de los casos la situación generada tras la Covid19 no ha afectado a la situación contractual $(88,1 \%)$. En el caso de la educación no formal, no se han visto afectados una tasa del $89,6 \%$ y del $71,1 \%$ en el de la educación formal. Entre quienes sí han sido afectados existen diferentes casuísticas: personas que han sufrido despidos o extinciones de contratos, expedientes temporales de regulación de empleo (ERTE) parciales o totales, expedientes de regulación de empleo (ERE), quienes han sido afectados por el permiso retribuido recuperable debido al estado de alarma, nuevas contrataciones por las necesidades de la crisis sanitaria y otras causas no incluidas entre las anteriores como bajas laborales por ser población de riesgo o reducciones por cuidado de menores recogidas en la normativa de conciliación laboral por la situación de crisis sanitaria. Recogemos los datos de manera exhaustiva en la figura 3.

Por tipo de empleador, quienes trabajan en el sector público no se han visto afectados contractualmente en un 92,7\% y sí se han visto en un 7,3\%. En el ámbito privado tasa de no afectados es de un $89,3 \%$ y la de los afectados en su contrato de un 10,7\%.

En relación con la afectación laboral, planteamos tres escenarios diferenciados: a) quienes no han sufrido afectación; b) quienes han sufrido modificaciones respecto a turnos, horarios, vacaciones, etc., y c) quienes se encuentran teletrabajando.

La mayoría de quienes trabajan en educación no formal señala el segundo escenario $(56,4 \%)$, sin embargo, el escenario tercero de teletrabajo es mayoritario entre quienes se engloban dentro de la educación formal $(75,9 \%)$. En la figura 4 recogemos detalladamente los diferentes escenarios. 


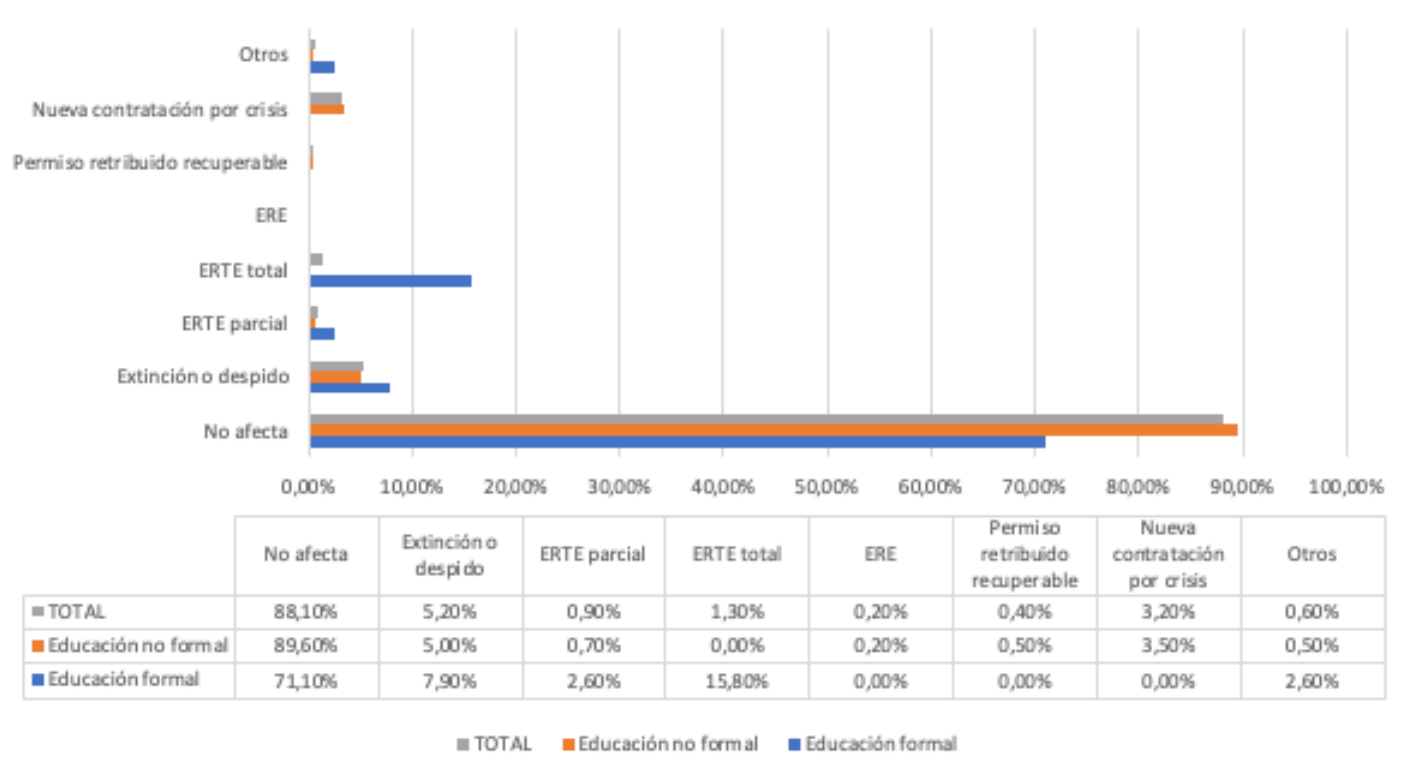

Figura 3. Repercusión en la situación contractual

Fuente: Elaboración propia.

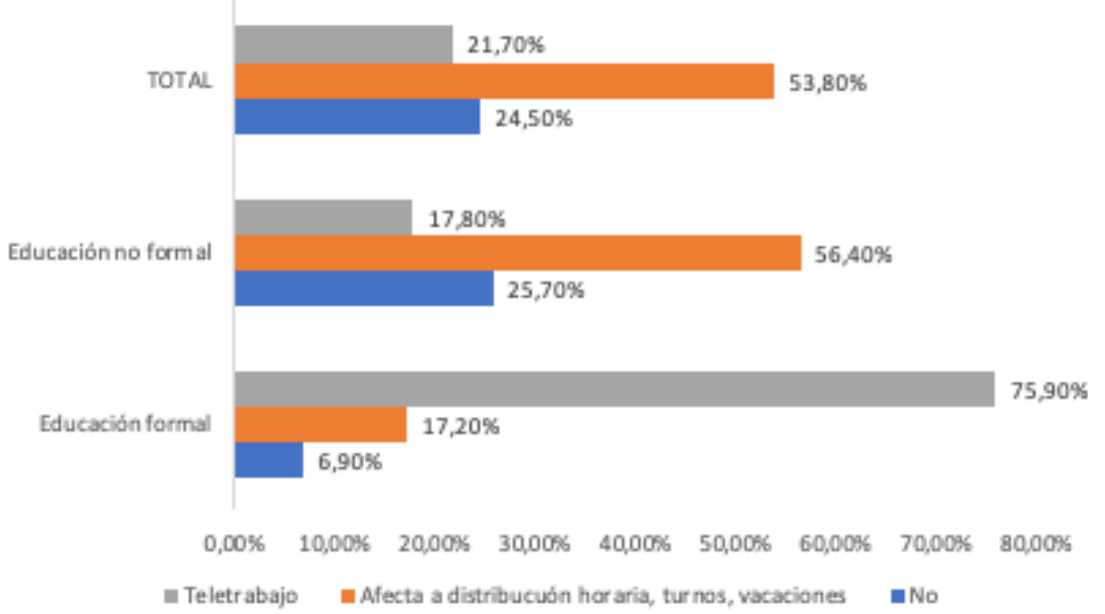

Figura 4. Repercusión en la situación laboral

Fuente: Elaboración propia.

Según tipo de empleador, quienes trabajan en el sector público no se han visto afectados laboralmente en un 20,5\%, han señalado el segundo escenario en un $43,4 \%$ y se encuentran teletrabajando en un 36,1\%. En el ámbito privado tasa de no afectados es de un 26,1\%, el segundo escenario con modificaciones de horarios, vacaciones, etc., lo señala un 57,7\% y teletrabajan en un $16,1 \%$.

Por último, se preguntó a las personas participantes si su actividad profesional había sido declarada como esencial dentro de las medidas tomadas por el gobierno. La respuesta ha sido positiva en un total del $82,7 \%$ de los casos y negativa en un $17,3 \%$. Presenta grandes variaciones entre quienes trabajan en la educación formal (declarada esencial en el 34,2\% de los casos) y quienes trabajan en educación no formal (declarada esencial en el $87 \%$ de los casos). 
No se ha encontrado relación significativa entre el tipo de empleado (público o privado con/sin ánimo de lucro y autoempleo) y afectación en la situación contractual (chi-cuadrado $=1,623 ; \mathrm{p}=0,203)$, pero sí entre en la afectación a la situación laboral (chi-cuadrado $=20,459 ; \mathrm{p}=0,000$ ).

\subsection{Carga de trabajo}

Se preguntó a las personas participantes sobre su carga de trabajo debido a la situación derivada de la crisis de la Covid-19, con tres opciones de respuesta: se ha mantenido igual que antes, ha aumentado o ha disminuido.

La mayoría de las personas participantes consideran que su carga de trabajo ha aumentado debido a la crisis. La tasa total entre quienes están realizando actividad presencial que indica un aumento es muy elevada, del 77,4\%; en el caso del teletrabajo, también el aumento es la opción más señalada, pero con bastante diferencia respecto al presencial, lo indica un $39,4 \%$ de la muestra total que teletrabaja. Únicamente en educación formal a través de teletrabajo no ha sido la opción más indicada. La opción de disminución de la carga de trabajo es señalada por un $5,7 \%$ en el caso del trabajo presencial y un $36,2 \%$ en el teletrabajo. Indicamos las tasas detalladas por ámbitos y situaciones laborales en la figura 5 .

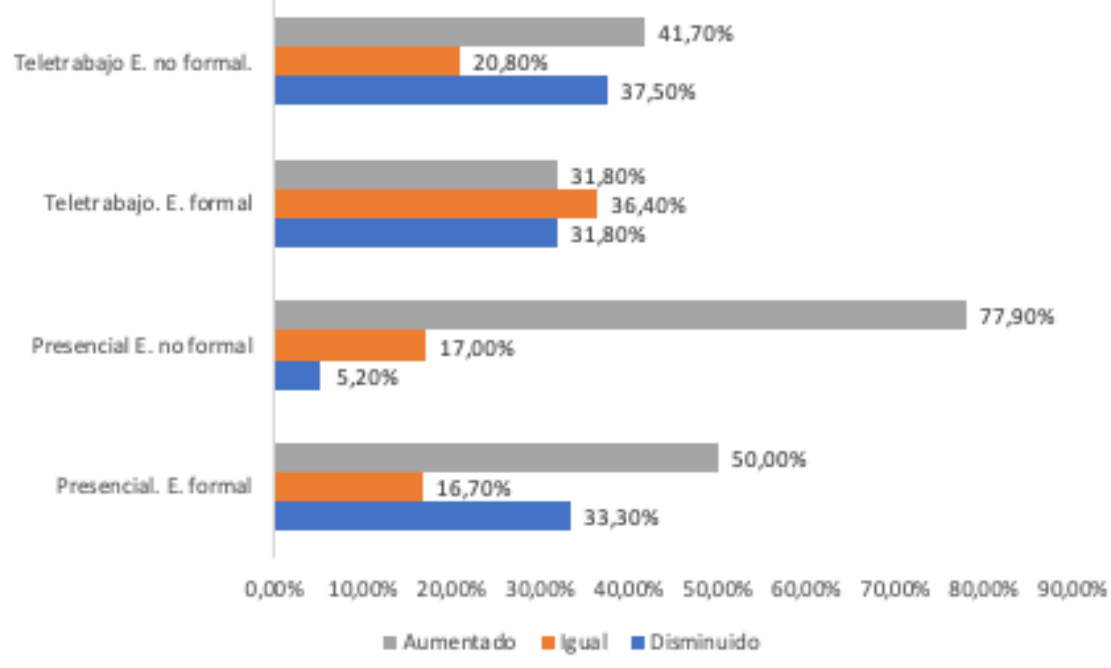

Figura 5. Repercusión en la carga de trabajo Fuente: Elaboración propia.

Se aprecia diferencia significativa en la carga de trabajo entre ámbito formal y no formal (chi-cuadrado $=17,318 ; \mathrm{p}=0,000$ ), habiendo aumentado notablemente en el caso de los profesionales del ámbito no formal.

\subsection{Cumplimiento de los objetivos planteados}

Las personas encuestadas fueron preguntadas acerca su consideración sobre la posibilidad de desarrollar de una manera correcta la actividad laboral para cumplir con los objetivos planteados en función los recursos disponibles en la actual situación (figura 6). Dentro del trabajo presencial la media indicada es de 2,9; la mediana y la moda de 3; el mínimo 1 y el máximo 5; con una desviación típica de 1,066. Entre quienes teletrabajan la media indicada es de 2,9; la mediana y la moda de 3; el mínimo 1 y el máximo 5; con una desviación típica de 1,066 . 
En el caso de la educación formal y no formal a través de teletrabajo, predominan los valores medios. Sin embargo, en la modalidad presencial, dentro de la educación formal, el grado de acuerdo es alto.

Los niveles inferiores de acuerdo (nulo y bajo) suman un tercio de los participantes en educación no formal de modo presencial (33,7\%) y un $29,1 \%$ en el caso del teletrabajo en este ámbito. Estos niveles se sitúan en $27,3 \%$ en el caso de la formal a través del teletrabajo y en el $14,3 \%$ en modo presencial.

Los valores elevados de acuerdo (alto y muy alto) son señalados en mayor medida en el caso de la educación formal de modo presencial (42,9\%), seguido por la educación no formal a través de teletrabajo (40,3\%), en tercer lugar, lo señalan quienes realizan teletratrabajo en educación formal y, en último lugar, quienes trabajan de manera presencial en educación no formal $(25 \%)$.

En educación formal presencial, casi un tercio de la muestra indica que no es de aplicación este ítem en su situación (28,6\%), mientras que en educación no formal lo indica un $2,8 \%$. No se aprecia diferencia significativa en la valoración de la posibilidad de consecución de objetivos entre ámbito formal y no formal (chi-cuadrado $=3,186 ; \mathrm{p}=0,203$ ).

Entre quienes trabajan de manera presencial, indica los valores inferiores una tasa del $34,3 \%$, los medios un $39,5 \%$ y los más elevados un $26,1 \%$. En el caso del teletrabajo, los valores más bajos son señalados por un $28,7 \%$, los medios por un 30,9\% y los más elevados en un 40,4\% de los casos. Se aprecia diferencia significativa en la valoración de la posibilidad de consecución de objetivos entre trabajo presencial y teletrabajo (chi-cuadrado $=7,247900 ; \mathrm{p}=0,0267$ ).

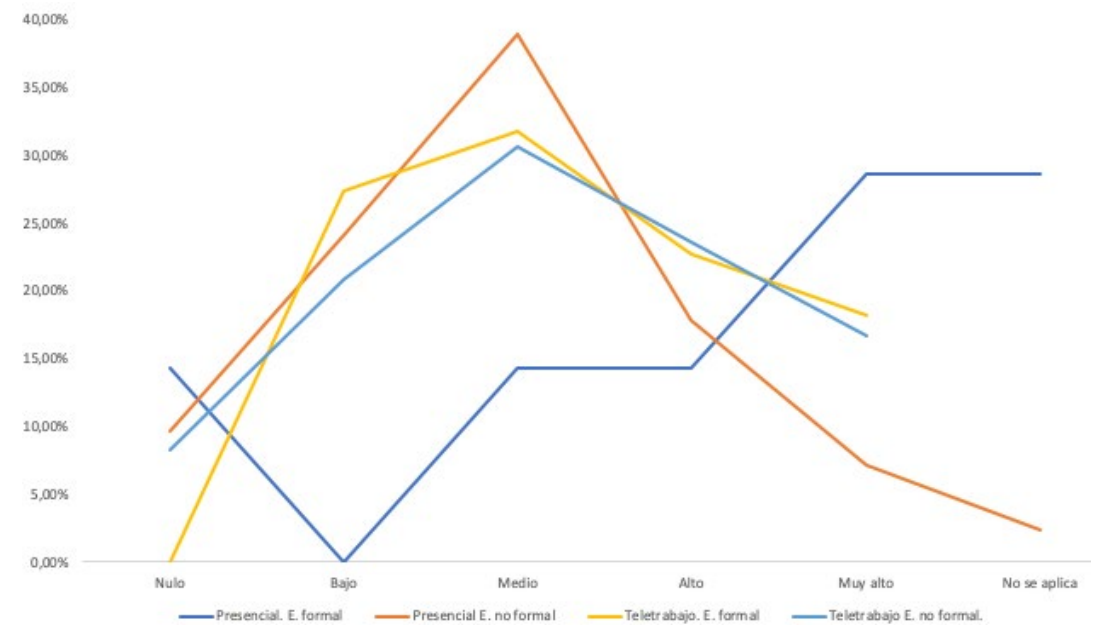

Figura 6. Posibilidad de cumplir con los objetivos planteados Fuente: Elaboración propia.

\subsection{Disponibilidad de herramientas y competencias digitales en usuarios para ser atendidos a través del teletrabajo}

Relacionado con el cumplimiento de los objetivos, se preguntó a las personas que estaban teletrabajando por la disponibilidad de herramientas telemáticas adecuadas entre los usuarios de su servicio para continuar su atención, así como por las competencias digitales de estos. 
En concreto se indicaba señalar su grado de acuerdo con las siguientes indicaciones:

- Los usuarios/as de mi servicio disponen de las herramientas suficientes para ser atendidos mediante esta modalidad.

- Los usuarios/as de mi servicio poseen competencia digital suficiente para ser atendidos mediante esta modalidad.

Respecto a la primera cuestión, la disponibilidad de herramientas entre los usuarios, la media es de 2,37; la mediana y la moda de 2. El mínimo indicado es de 1 y máximo de 5 con una desviación típica de 1,047. Los niveles inferiores de acuerdo (nulo o bajo) ocupan la mayor tasa en educación formal (50\%) y educación no formal (62,5\%) y los niveles superiores (alto o muy alto) apenas suman un 13,6\% en educación formal y un 15,8\% en educación no formal.

En relación con la segunda cuestión, las competencias digitales para recibir este tipo de atención, la media es de 2,39; la mediana y la moda de 2. El mínimo indicado es de 1 y máximo de 5 con una desviación típica de 1,0597. De nuevo los niveles más bajos de acuerdo suman una mayor tasa tanto en educación formal (50\%) como en no formal (68,1\%). Los niveles superiores acumulan un 22,7\% en educación formal y un 15,3\% en educación no formal. Reflejamos los diferentes datos en el cuadro1.

No se encuentran diferencias significativas en la valoración de la disponibilidad de herramientas entra ambos ámbitos (chi-cuadrado $=1,792 ; \mathrm{p}=0,408$ ), ni la valoración de la competencia digital de los usuarios (chi-cuadrado $=2,400 ; \mathrm{p}=0,301$ ).

Cuadro 1. Herramientas y competencias digitales en usuarios para la teleatención

\begin{tabular}{lccccc}
\hline & NULO & BAJO & MEDIO & ALTO & MUY ALTO \\
\hline Disponibilidad de herramientas & & & & & \\
Educación formal & $18,20 \%$ & $31,80 \%$ & $36,40 \%$ & $9,10 \%$ & $4,50 \%$ \\
Educación no formal & $22,20 \%$ & $40,30 \%$ & $22,20 \%$ & $12,50 \%$ & $2,80 \%$ \\
Competencias digitales & & & & & \\
Educación formal & $22,70 \%$ & $27,30 \%$ & $27,30 \%$ & $13,60 \%$ & $9,10 \%$ \\
Educación no formal & $16,70 \%$ & $51,40 \%$ & $16,70 \%$ & $12,50 \%$ & $2,80 \%$ \\
\hline
\end{tabular}

Fuente: Elaboración propia.

\subsection{Satisfacción laboral}

También se encuestó a los participantes sobre su grado de satisfacción laboral actual. La media general de satisfacción es ligeramente mayor entre quienes trabajan de manera presencial $(2,97)$ que entre quienes teletrabajan $(2,86)$. En el caso del trabajo presencial la mediana y moda son de 3 , con un mínimo 1 y un máximo de 5 y una desviación típica de 1,083. Entre quienes teletrabajan, mediana y moda son también de 3, un mínimo 1 y un máximo de 5, con una desviación típica de 0,968.

Por sectores, entre quienes trabajan en educación formal de manera presencial ninguna persona indica los valores inferiores (nulo o bajo) y los valores superiores (alto y muy alto) acumulan una tasa del $80 \%$. No es así entre quienes teletrabajan en educación formal donde la mayor tasa se concentra en niveles medios $(36,1 \%)$.

En el caso de la educación no formal, quienes siguen trabajando de manera presencial indican los niveles más bajos en un 36,3\%, los superiores en un 32,4\% y el nivel intermedio en un $36,1 \%$. Entre quienes trabajan en educación no formal aumenta la tasa de acuerdo con el nivel intermedio hasta el 40,9\%, disminuyen los inferiores hasta $27,2 \%$ y los 
superiores descienden ligeramente al $31,8 \%$. En la figura 7 exponemos detalladamente las respuestas.

No se encuentran diferencias significativas en la valoración de la disponibilidad de la satisfacción laboral entre educación formal y no formal (chi-cuadrado $=2,336 ; p=0,311$ ).

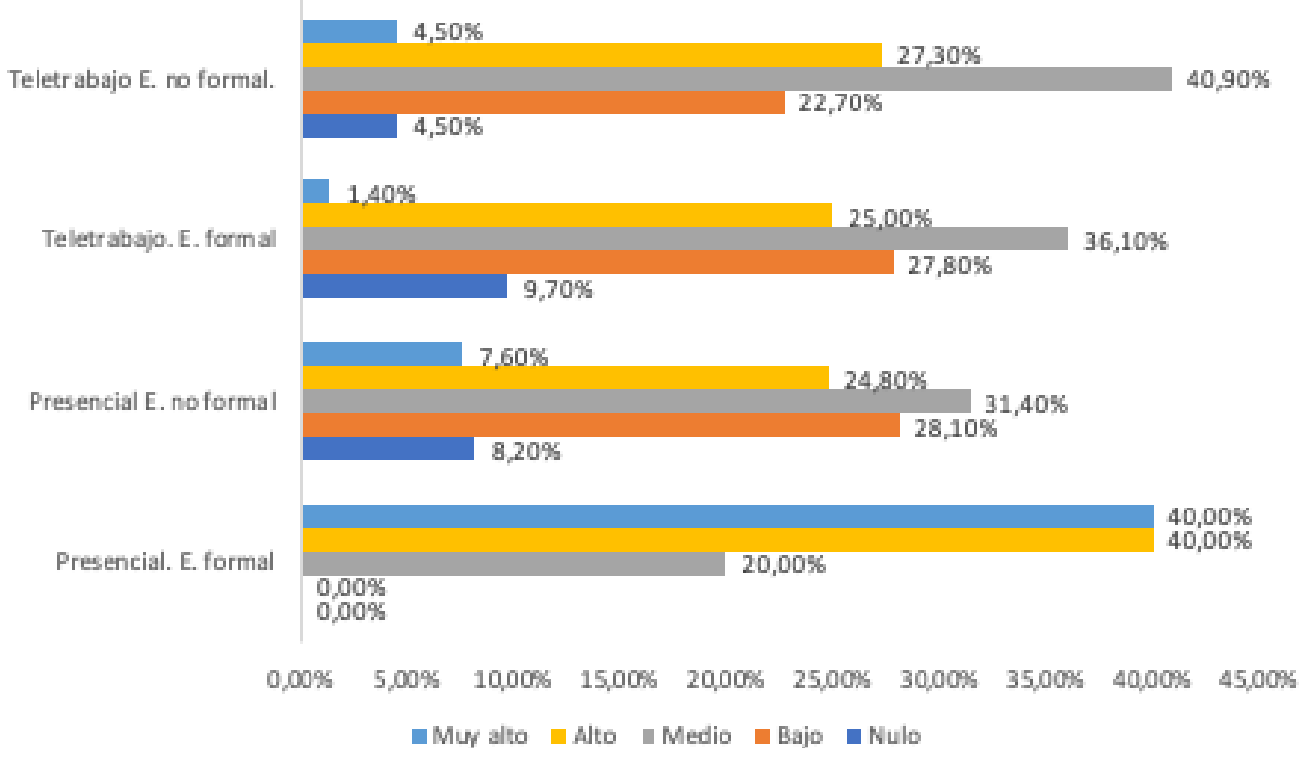

Figura 7. Satisfacción laboral de los educadores/as sociales que trabajan con menores en situación de la Covid-19

Fuente: Elaboración propia.

\subsection{Necesidad de recursos o formación}

Se formuló a los participantes la pregunta abierta, de carácter opcional, sobre si consideraba necesario o echaba en falta algún tipo de formación o recurso que pudiera ser de utilidad para realizar su trabajo ante este tipo de situaciones. De entre los participantes que respondieron, 14 lo hicieron únicamente con un sí y 27 con un no. Entre quienes desarrollaron su respuesta hemos elaborado las categorías principales (cuadro 2). Los recursos, la formación, los EPIs y los protocolos son los que más respuestas suscitaron, con bastante diferencia respecto al resto de categorías. A continuación, analizamos los principales aspectos y destacamos algunas de las opiniones de las personas participantes.

En lo referido a formación existen demandas genéricas de formación y también en aspectos psicológicos o medidas de higiene y prevención entre quienes trabajan de manera presencial; entre aquellas personas que realizan teletrabajo las demandas están más relacionadas con la competencia digital, herramientas y plataformas para realizar su actividad laboral.

Formación en plataformas digitales y herramientas similares. (Mujer. Cataluña. 16 años de experiencia)

Falta formación, protocolos claros. (Hombre. Aragón. 20 años experiencia)

Refuerzo personal, para formación online de los menores. (Mujer. Castilla La Mancha. 20 años de experiencia)

La formación e información han sido inexistentes. (Hombre. País Vasco. 29 años experiencia) 
La formación que se recibe es nula, pasa de un día para otro a teletrabajar, en el que tienes que ir improvisando y adecuando tus medios. (Mujer. Cantabria. 14 años de experiencia)

Cuadro 2. Demandas de recursos y formación

\begin{tabular}{lccc}
\hline & PRESENCIAL & TELETRABAJO & TOTAL \\
\hline Sí & 13 & 1 & 14 \\
No & 19 & 8 & 27 \\
Recursos & 16 & 13 & 29 \\
Formación & 16 & 12 & 28 \\
EPIs & 17 & 0 & 17 \\
Protocolos & 13 & 0 & 13 \\
Información & 8 & 1 & 9 \\
Emocional & 8 & 1 & 9 \\
Desprevenidos & 3 & 0 & 3 \\
Correcto & 3 & 0 & 3 \\
\hline
\end{tabular}

Fuente: Elaboración propia.

Las demandas sobre recursos entre quienes trabajan presencialmente están relacionadas principalmente con recursos humanos de apoyo para la atención, así como sobre algunos recursos materiales, entre los que se encuentran recursos informáticos para continuar con la actividad de los usuarios jóvenes. Entre quienes teletrabajan se recogen demandas referidas a recursos informáticos y digitales, así como herramientas para los usuarios.

Más recursos profesionales, estamos saturadas sin apoyo alguno. (Mujer. Andalucía. 4 años de experiencia)

Faltan recursos informáticos para realizar las clases telemáticas. (Mujer. Extremadura. 16 años de experiencia)

Hay situaciones vividas con los menores que hubieran necesitado apoyo del personal específico para el cumplimiento del confinamiento con adolescentes. (Mujer. Extremadura. 13 años de experiencia)

Los EPIs son una demanda entre los trabajadores presenciales, algunos de los participantes indican que en las primeras semanas eran muy escasos o nulos. Lo mismo ocurre respecto a los protocolos, que son también demandados por los profesionales que realizan su labor de manera presencial.

En mi caso, las tres primeras semanas no habia EPIS. $Y$ estaba prohibido ir a tu puesto de trabajo con ellas aun siendo tuyas. (Mujer. País Vasco. 14 años de experiencia)

Falta de EPI, algunos compañeros no usan guantes ni mascarillas, debería ser obligatorio. (Mujer. Cataluña. 20 años de experiencia)

La Mutua no se ha hecho cargo de nada respecto al tema de seguridad laboral. No nos ha proporcionado material de protección. Ni nada. (Mujer. País Vasco. 13 años de experiencia.)

Los participantes reclaman información clara y se quejan de la poca información recibida respecto a la crisis y como los usuarios reclaman información que no pueden proporcionar. A continuación, reproducimos unas respuestas que resumen las demandas en este aspecto y otros anteriores:

Necesitamos EPIs y cómo usarlos. $Y$ los menores demandan información que no sabemos resolver. (Mujer. Galicia. 4 años de experiencia)

Sí, medidas de prevención y protocolos de actuación específicas, más apoyo por parte de la entidad, y de la administración competente tanto en equipo como en recursos 
humanos ya que las ratios de personal son insuficientes... (Hombre. Castilla LaMancha. 22 años de experiencia)

Protocolos de actuación claros. (Mujer. Castilla y León. 3 años de experiencia)

Instrucciones claras consensuadas con sanidad de aplicación específica en recursos de protección. (Mujer. Castilla La-Mancha. 12 años de experiencia)

La referencia a la carga emocional y el necesario apoyo emocional y psicológico es otra de las respuestas más comunes, dirigida tanto a profesionales como a los menores y familias.

Estamos parcheando la situación y la carga laboral y emocional es mayor. (Mujer.

Canarias. 7 años de experiencia)

Echo en falta recursos de acompañamiento emocional. (Mujer. Comunidad Valenciana. 15 años de experiencia)

Como afrontar situaciones de extrema emergencia, apoyo emocional a familias en riesgo. (Mujer. Comunidad de Madrid. 20 años de experiencia)

Apoyo psicológico a menores. (Mujer. Aragón. 12 años de experiencia)

Control de stress, ansiedad... (Hombre. Andalucía. 10 años de experiencia)

Otros temas que captan la atención son las opiniones sobre como la crisis ha encontrado desprevenidos a los servicios de atención. Asimismo, algunos usuarios consideran correctos tanto la formación como los recursos.

\section{Discusión y conclusiones}

A través de nuestro estudio realizamos un dibujo del impacto de la crisis sanitaria durante el periodo de la segunda quincena de abril de 2020, entre los y las profesionales de la acción socioeducativa que realizan su intervención con menores desde el ámbito formal y no formal. Recordamos que era uno de los momentos más graves del desarrollo de la pandemia en España, con más de 200.000 casos confirmados y casi 16.000 defunciones.

La crisis de la Covid-19 ha tenido importantes repercusiones en los sectores sanitarios, políticos, sociales y económicos en todo el mundo. El ámbito de la educación ha sido uno de los grandes afectados a través de las medidas decretadas (UNICEF, 2020; UNESCO, s.f.) que en España ha supuesto la paralización de las actividades presenciales no esenciales a través de los RD 463/2020 y RDL 10/2020 y que, en la práctica, ha supuesto la adaptación nuevas formas de trabajo y de relación en el ámbito de la educación.

En el ámbito socioeducativo, acostumbrado a las intervenciones presenciales en las que la relación personal es una técnica habitual, las TIC aún no están firmemente consolidadas como herramienta de trabajo. Aspectos como la falta de dispositivos o de competencia digital se han manifestado en nuestro estudio, en línea a lo aportado por otros trabajos que recogen la brecha digital por motivos económicos o sociales en el acceso a los dispositivos, o por desconocimiento de uso (de Rosa, 2017; Planella y Martínez, 2010).

Aunque hablamos de infancia y juventud, un colectivo generalmente adscrito al concepto de nativo digital, en los últimos tiempos se está poniendo en duda este término debido a que la utilización rutinaria de los dispositivos no, necesariamente, implica el desarrollo de la competencia digital y el uso adecuado de los instrumentos tecnológicos como habitualmente se esperaría de un nativo digital (Kirschner y Bruyckerec, 2017; PérezEscoda, Castro-Zubizarreta y Fandos-Igado, 2016) Ante el cierre de las escuelas UNESCO (s.f.) apunta diversos aspectos que pueden tener consecuencias negativas, como 
el acceso desigual a las plataformas de teleformación o la falta de preparación de los padres para la enseñanza a distancia.

Entre los profesionales que teletrabajan, las demandas se dirigen a la falta de equipos tecnológicos, algo que tampoco es ajeno a otros sectores educativos donde se ha tenido que desarrollar el trabajo con medios propios.

Otro aspecto relevante derivado del estudio es la disminución de la percepción de la satisfacción laboral respecto a trabajos anteriores (Martínez-Pérez y Lezcano-Barbero, 2020). Asimismo, se ha detectado un porcentaje elevado de profesionales que consideran que su carga de trabajo ha aumentado como consecuencia de la pandemia, así como demandas de recursos humanos y materiales y de protocolos de información y actuación claros. La demanda de EPIs es especialmente relevante teniendo en cuenta el momento de la recogida de datos.

La carga de trabajo y las prácticas, políticas de empresa y de gestión de personal repercuten en aspectos como la calidad del servicio pudiendo derivar en problemas como el absentismo (Acker, 2018; Calitz, Roux, y Strydom, 2014; Gómez, Alonso y Llamazares, 2018; Van Aerden et al., 2016). UNICEF (2020) recogía como algunas de las consecuencias derivadas de la pandemia pueden ser el retraso de expedientes e informes y como la gestión de los recursos humanos puede afectar al rendimiento.

En nuestro trabajo se manifiestan también demandas de apoyo psicológico y emocional y consecuencias de la carga de trabajo y la situación vivida como el estrés o la ansiedad. Hemos recogido estudios que muestran las posibles consecuencias psicológicas de la pandemia entre la población ( $\mathrm{Li}$ e al., 2020) y, especialmente, entre los menores (Liang et al., 2020). También como otro de los posibles peligros derivados de la crisis de la Covid19 puede ser un repunte de la violencia intrafamiliar (Gyamfi y Jackson, 2020; UNICEF, 2020; Usher et al., 2020).

Ante esta situación parecen necesarias, como apuntan Liang y otros (2020), intervenciones en salud mental adecuadas para los diferentes grupos; también entre los profesionales. Acudir a diario al centro de trabajo ante situaciones complicadas, con falta de recursos y ver el día a día de los menores sin poder resolver las dudas y cuestiones de estos puede derivar en situaciones complicadas que deben ser atendidas.

A nivel contractual, al ser declarados en la mayoría de los casos actividad esencial, no ha habido una afectación demasiado elevada, aunque sí encontramos tres puntos de diferencia entre el sector público y privado. Fullana y otros (2007) alertan sobre las políticas sociales actuales que tienden a desvincular los servicios sociales de las prestaciones públicas, con consecuencias como la precarización del sector a través de la subcontratación y otras prácticas que revierten en la calidad del servicio. La afectación aumenta en el caso laboral, con modificaciones de horarios y turnos o pasar a la situación de teletrabajo.

Otro aspecto relevante de nuestro trabajo es que la percepción del grado de cumplimiento de los objetivos educativos entre los profesionales no es excesivamente optimista, la media se sitúa en 2,9. No disponemos de estudios previos para comparar esta percepción, no obstante consideramos que esta situación no debería prolongarse en el tiempo pues, de lo contrario, la efectividad de los programas se verá seriamente comprometida con importantes consecuencias para los menores y la sociedad en su conjunto. 
En síntesis, en esta situación, en que los escenarios varían muy rápidamente, comprendemos que es difícil establecer protocolos complejos y adecuados a cada realidad, pero precisamente por esto, es necesario que la información fluya rápido y de una manera clara y transparente desde quienes programan hacia quienes deben implementar las actuaciones.

Estamos de acuerdo con UNICEF (2020) cuando manifiesta que no debemos olvidar que en ante una emergencia "los derechos no desaparecen, ni se apartan, ni se posponen, al contrario: es necesario hacer un esfuerzo adicional por garantizarlos" (p. 4) que en el ámbito socioeducativo implicará, al menos, dotar de medios humanos y materiales a los equipos de atención y de herramientas y competencias a los propios menores.

No debemos olvidar que nuestro trabajo muestra la realidad de un sector de profesionales que, en la mayoría de los casos, trabajan con menores en situación de dificultad como se observa a través de la muestra, donde más de un $85 \%$ desarrolla su labor en recursos de tutela o reforma del ámbito de la infancia y la juventud. UNESCO (s.f.) recoge aspectos sociales en los que puede incidir el cierre de las escuelas: aumento del absentismo, desigualdad ante el cuidado de los niños, costos económicos para las familias e, incluso, carencias en la alimentación entre los menores.

Por último, apuntar, tal y como puede observarse en la muestra, que el número de educadores y educadoras sociales integrados en centros educativos es aún escaso. Sin embargo, varios de los riesgos planteados por UNICEF (2020) y UNESCO (s.f.) derivados de la pandemia como el absentismo y el abandono escolar, la estigmatización y el aumento de la violencia, la atención a la vulnerabilidad y la gestión de recursos y coordinación con los servicios sociales, son algunas de las funciones realizadas por estos profesionales en aquellas comunidades autónomas en que realizan su trabajo en la escuela (Ruíz-Román, Molina y Alcaide, 2019; Sierra et al., 2017; Vila, Cortés y Martín, 2020).

De nuestro estudio, podemos extraer algunas conclusiones:

- Los educadores y educadoras sociales que trabajan con menores son en su mayoría mujeres y la prestación de servicios de acción socioeducativa con menores se lleva a cabo, principalmente, a través del sector privado (tercer sector sin ánimo de lucro o empresa privada con ánimo de lucro) concertado con la administración y en menor medida a través de la gestión directa.

- La satisfacción laboral de este sector en la actual crisis sanitaria es menor que la media de satisfacción general del colectivo.

- Los profesionales demandan mayor dotación de recursos humanos y materiales, así como protocolos de actuación e información y formación para llevar a cabo su labor.

- Es necesario plantearse la relación entre TIC y educación social como una herramienta más en la intervención socioeducativa. Quizá sea necesario invertir recursos en el equipamiento y la formación necesaria (entre profesionales y usuarios) para optimizar las posibilidades que ofrecen.

- Parece evidente que la pandemia va a tener consecuencias en la salud, especialmente psicológicas, que se podrían extender más que la duración del virus. Por ello es necesario plantear recursos de apoyo para colectivos 
vulnerables y para los profesionales que han estado realizando procesos de atención en primera línea durante la pandemia.

- Urge que los responsables educativos planteen un debate serio sobre la inclusión de la figura del educador/a social en los centros educativos de todo el Estado. Situaciones como la pandemia o la próxima aprobación de la ley de protección a la infancia y adolescencia ponen de manifiesto las posibilidades de esta figura en la escuela para tratar aspectos sociales y actuar como agente vertebrador de las relaciones entre escuela y los recursos sociales.

Dentro de las limitaciones del estudio, debemos manifestar las propiciadas, también, por la situación excepcional en que nos encontramos. La literatura científica es aún escasa y no se disponen de herramientas validadas para la medición. No obstante, consideramos que aportamos datos de valor para avanzar en el conocimiento de la repercusión de la Covid-19 entre los profesionales y los usuarios de los servicios de atención a menores en nuestro país. Respecto a la muestra, la referida al ámbito no formal es lo suficientemente amplia, pero entre quienes trabajan en educación reglada el número es reducido, en consonancia con la realidad profesional de la educación social (ANECA, 2005), por lo que convendría interpretar los datos relacionados con los profesionales de la educación formal con prudencia.

Deseamos finalizar transmitiendo dos lecciones que hemos (deberíamos haber) visualizado con claridad:

- La salud no es una variable individual. Si nuestro entorno no se encuentra bien, nosotros tampoco podremos estarlo. Este pensamiento no es nuevo, se propuso ya en 1948 en la fundación de la OMS. Ver diariamente el número de muertos, a pesar de que no les conociéramos, ha sido extremadamente doloroso para todos.

- Estar preparados para responder a situaciones complejas, no sólo pandemias, debe estar programado, planificado, organizado y presupuestado, especialmente con los más vulnerables. O volveremos a tropezar con la misma piedra.

\section{Referencias}

Acker, G. M. (2018). Self-care practices among social workers: Do they predict job satisfaction and turnover intention? Social Work in Mental Health, 16(6), 713-727. https://doi.org/10.1080/15332985.2018.1494082

Álvarez Fernández, A. M. (2017). El desempeño profesional del educador y la educadora social: Funciones, competencias y creencias de autoeficacia (Tesis doctoral). Universidad Autónoma de Madrid, España.

ANECA. (2005). Libro blanco de grado en pedagogía y educación social. ANECA.

Cacho Labrador, X. (1998). L'educadora i l'educador social a Catalunya. APESC.

Calderón, M. J. (2013). La profesión de la educación social en Europa. Estudio comparado. CGCEES.

Calitz, T., Roux, A. y Strydom, H. (2014). Factors that affect social workers' job satisfaction, stress and burnout. Social Work/Maatskaplike Werk, 50(2), 153-169.

https://doi.org/10.15270/50-2-393

Centro Nacional de Epidemiología. (2020). Informe sobre la situación de Covid-19 en España. Informe Covid-19 no 27. ISCIII. 
Creswell, J. W. (2012). Educational research. Planning, conducting, and evaluating quantitative and qualitative research. Pearson.

Creswell, J. W. (2015). A concise introduction to mixed methods research. Sage.

de Rosa, E. (2017). Social innovation and ICT in social services: European experiences compared, Innovation. The European Journal of Social Science Research, 30(4), 42 1-432. https://doi.org/10.1080/13511610.2017.1348936

Fernández, G. (30 de marzo de 2020). Tensión y miedo entre los menores tutelados por no saber qué ocurrirá con ellos tras la pandemia. El cierre digital. https://elcierredigital.com/investigacion/168631550/menores-tutelados-covid-ocurriradespues-pandemia.html

Fullana, J., Pallisera, M., Tesouro, M. y Castro, M. (2007). La inserción laboral de los diplomados en educación social: Análisis de las características de los procesos de inserción y reconocimiento profesional. Bordón, 59(4), 565-580.

Galán Carretero, D. (2019). La realidad de los educadores sociales en el estado español. Experiencia evolutiva en los centros de educación secundaria de Extremadura. Educación Social: Revista de Intervención Socioeducativa, 71, 79-104.

Gómez, R., Alonso, M. y Llamazares, M. L. (2018). Evaluation of job satisfaction in a sample of Spanish social workers through the 'job satisfaction survey’ scale. European Journal of Social Work, 21(1), 140-154. https://doi.org/10.1080/13691457.2016.1255929

Isaifan, R. (2020). The dramatic impact of Coronavirus outbreak on air quality: Has it saved as much as it has killed so far? Global Journal of Environmental Science and Management, 6(3), 275-288.

Kirschner, P. A. y Bruyckerec, P. D. (2017). The myths of the digital native and the multitasker. Teaching and Teacher Education, 67, 135-142. https://doi.org/10.1016/j.tate.2017.06.001

Li, S., Wang, Y., Xue, J., Zhao, N. y Zhu, T. (2020). The impact of covid-19 epidemic declaration on psychological consequences: a study on active weibo users. International Journal of Environmental Research and Public Health, 17(6), 20-32. https://doi.org/10.3390/ijerph 17062032

Liang, L., Ren, H., Cao, R., Hu, Y., Qin, Z., Li, C. y Mei, S. (2020). The effect of covid-19 on youth mental health. Psychiatric Quarterly, in press. https://doi.org/10.1007/s 11 126-020-09744-3

López Noguero, F. (2005). La educación social especializada con personas en situación de conflicto social. Revista de Educación, 336, 57-71.

López Zaguirre, R. (2013). Las educadoras y los educadores sociales en los centros escolares en el estado español. Revista de Educación Social, 16, 1-6.

Martínez-Pérez, A. y Lezcano-Barbero, F. (2020). Satisfacción profesional de los educadores sociales: Factores de influencia. Bordón, en prensa. https://doi.org/10.13042/Bordon.2020.72172

Médicos del Mundo. (2020). Prevención y control del Covid-19 en un centro para menores no acompañados en Melilla. https://www.medicosdelmundo.org/actualidad-ypublicaciones/noticias/prevencion-Covid-19-menores-extranjeros-solos-Melilla

Ministerio de Sanidad. (2020). Buenas prácticas en los centros de trabajo. https://www.mscbs.gob.es/gabinetePrensa/notaPrensa/pdf/GUIA1 10420172227802.pdf 
Ortega, J., Caride, J. A. y Úcar, X. (2013). La pedagogía social en la formación-profesionalización de los educadores y las educadoras sociales, o de cuando el pasado construye futuros. Revista de Educación Social, 17, 1-24.

Pérez-Escoda, A., Castro-Zubizarreta, A. y Fandos-Igado, M. (2016). Digital skills in the Z generation: Key questions for a curricular introduction in Primary School. Comunicar, 49, 71-79. https://doi.org/10.3916/C49-2016-07

Pérez Serrano, G. (2003). Pedagogía social, educación social. Construcción científica e intervención práctica. Narcea.

Planella, J. y Martínez, O. (2010). Pedagogía y tecnología de la esperanza. La educación social y las nuevas formas tecnológicas de acompañamiento. Revista de Educación Social, 11, 57-65.

Ruíz-Román, S., Molina Cuesta, L. y Alcaide Vives R. (2019). We have a common goal: Support networks for the educational and social development of children in disadvantaged areas. The British Journal of Social Work, 49(6), 1658-1676. https://doi.org/10.1093/bjsw/bcz063

Sierra, J. E., Vila, E. S., Caparrós, E. y Martín, V. M. (2017). Rol y funciones de los educadores y las educadoras sociales en los centros educativos andaluces. Análisis y reflexiones. Revista Complutense de Educación, 28(2), 479-495. https://doi.org/10.5209/rev_RCED.2017.v28.n2.49542

Suanes, M. D. (2019). Diseño del perfil competencial de la profesión de la educación social (Tesis doctoral). Universidad de Córdoba, España.

Taber, K. S. (2017). The use of cronbach's alpha when developing and reporting research instruments in science education. Research in Science Education, 48, 1273-1296. https://doi.org/10.1007/978-94-6300-749-8

Tiana, A. (2014). La evolución de la educación social como campo académico y profesional. En A. Tiana, M. Somoza y A. M. Badanelli (Eds.), Historia de la educación social (pp. 21-56). UNED.

UNESCO. (s.f.). Consecuencias negativas del cierre de las escuelas. https://es.unesco.org/covid19/educationresponse/consecuencias

UNICEF. (2020). Covid-19: Proteger la salud en las aulas. Principios básicos para asegurar el derecho a la salud al reabrir los centros educativos.

https://www.unicef.es/sites/unicef.es/files/educa/unicef-educa-covid 19-aperturacentros-educativos-funcionamiento-seguro-2.pdf

Usher, K., Bhullar, N., Durkin, J., Gyamfi, N. y Jackson, D. (2020). Family violence and COVID19: Increased vulnerability and reduced options for support. International Journal of Mental Health Nursing, 29(4), 549-552. https://doi.org/10.1111/inm.12735

Vallés Herero, J. (2011). Análisis y valoración de las funciones de los educadores sociales en España (Tesis doctoral). Universidad Nacional de Educación a Distancia, España.

Van Aerden, K., Puig-Barrachina, V., Bosmans, K. y Vanroelen, C. (2016). How does employment quality relate to health and job satisfaction in Europe? A typological approach. Social Science Eं Medicine, 158, 132-140. https://doi.org/10.1016/j.socscimed.2016.04.017

Ventosa, V. J. (1989). Niveles formativos en la C.E.E: Correspondientes a las profesiones españolas en educación social. En VVAA., Actas del Congreso sobre la Educación Social en España (pp. 6374) Servicio de publicaciones del Ministerio de Educación y Ciencia.

Vila, E., Cortés, P. y Martín, V. (2020). los educadores y educadoras sociales en los centros educativos de Andalucía: Perfil y desarrollo profesional. REICE. Revista Iberoamericana sobre Calidad, Eficacia y Cambio en Educación, 18(1), 47-64.

https://doi.org/10.15366/reice2020.18.1.003 


\section{Breve CV de los autores}

\section{Alejandro Martínez-Pérez}

Licenciado en Pedagogía (UBU), Diplomado en Educación Social (UBU) y Máster en Educación y Tecnología: contenidos y estrategias digitales (UNED). Profesor Asociado, área de Didáctica y Organización Escolar. Departamento Ciencias de la Educación (Universidad de Burgos). Miembro del Grupo de Investigación EDINTEC. Doctorando del programa de educación de la UBU, realiza su tesis sobre TIC y educación social. Realiza actividad profesional externa a la universidad como orientador sociolaboral con jóvenes. ORCID ID: https://orcid.org/0000-0003-2800-505X. Email: alejandromp@ubu.es

\section{Fernando Lezcano-Barbero}

Licenciado en Ciencias de la Educación por la UNED y Doctor por la Universidad de Burgos. Profesor Titular de Universidad en el Área de Didáctica y Organización Escolar en la Universidad de Burgos. Miembro del Grupo de Investigación Reconocido: EDINTEC. Cuenta con un sexenio de investigación vivo. Desarrolla la investigación en el ámbito de la educación social y educativa vinculada a colectivos sociales desfavorecidos. ORCID ID: https://orcid.org/oooo-0001-7866-071X. Email: flezcano@ubu.es 